\title{
The clinicopathological significance of angiogenesis in hindgut neuroendocrine tumors obtained via an endoscopic procedure
}

Yoichiro Okubo ${ }^{1 *}$, Osamu Motohashi ${ }^{2}$, Norisuke Nakayama ${ }^{2}$, Ken Nishimura ${ }^{2}$, Rika Kasajima ${ }^{3}$, Yohei Miyagi ${ }^{3}$, Manabu Shiozawa ${ }^{4}$, Emi Yoshioka ${ }^{1}$, Masaki Suzuki ${ }^{1}$, Kota Washimi ${ }^{1}$, Kae Kawachi ${ }^{1}$, Madoka Nito ${ }^{5}$,

Yoichi Kameda ${ }^{1}$ and Tomoyuki Yokose ${ }^{1}$

\begin{abstract}
Background: As the World Health Organization grading system for gastroenteropancreatic-neuroendocrine tumors (GEP-NETs) may not always correlate with tumor progression, it is imperative that other independent predictors of tumor progression be established. To identify such predictors, we conducted a retrospective histopathological study of hindgut NETs, obtained from endoscopic procedures, and used statistical analyses to evaluate predictive factors.

Methods: We first obtained clinicopathological data of cases of hindgut NETs. Tissue sections from tumor samples were prepared and subjected to pathological examination. In particular, we calculated the microvessel density (MVD) and lymphatic microvessel density (LMVD) values, and performed appropriate statistical analyses.

Results: A total of 42 cases of hindgut NETs were selected for the study, 41 from the rectum and 1 from the sigmoid colon. Based on the Ki-67 labeling index, 34 cases were classified as NET G1 tumors and 8 as NET G2 tumors. MVD values ranged from $1.4 / \mathrm{mm}^{2}$ to $73.9 / \mathrm{mm}^{2}$ and $L M V D$ values from $0 / \mathrm{mm}^{2}$ to $22.9 / \mathrm{mm}^{2}$. MVD and LMVD were identified as risk factors for venous and lymphatic invasion of hindgut NETs. Moreover, MVD positively correlated with the maximum diameter of the tumor.

Conclusions: Tumor progression of NETs may cause angiogenesis and lymphangiogenesis, via an unknown mechanism, as well as lymphovascular invasion. Angiogenesis likely plays an important role in occurrence and progression in the initial phase of hindgut NETs.
\end{abstract}

Keywords: Neuroendocrine tumor, Hindgut, Microvessel, Angiogenesis

\section{Background}

Although previous investigators have reported on the prognostic value of lymphovascular invasion in several types of neoplasms, the results of these studies are somewhat controversial with regards to gastroenteropancreatic neuroendocrine tumors (GEP-NET) [1-3]. However, a number of recent studies have reported a higher than expected rate of venous and/or lymphatic invasion (lymphovascular invasion) in GEP-NET samples

\footnotetext{
* Correspondence: yoichiro0207@hotmail.com

'Department of Pathology, Kanagawa Cancer Center, 2-3-2, Nakao, Asahi-Ku, Yokohama, Kanagawa 241-8515, Japan

Full list of author information is available at the end of the article
}

obtained via endoscopic procedures [4-6]. In a case series from our own institution, we identified a lymphovascular invasion rate of approximately $30 \%$ for GEPNETs. Therefore, we conducted this study to specifically evaluate the lymphovascular status of GEP-NETs.

GEP-NETs have traditionally been regarded as relatively rare neoplasms [7]. However, recent surveillance data have shown the incidence and prevalence of GEPNETs to be higher than previously expected, likely due to recent technical advances in endoscopic and imaging examinations [8]. In Japan, the rectum is the most frequent site of GEP-NETs [8], as well as the second most common site in Western countries [4]. 
The grading system for GEP-NETs was updated by the World Health Organization (WHO) in 2010 [9-11]. This system is based only on the proliferative activity of tumor cells, as measured by the number of mitotic cells confirmed per 10 high-power fields (HPFs) and/or by the percentage of tumor cells showing positive immunoreactivity for the Ki-67 antigen (the Ki-67 labeling index). However, the proliferative characteristics of tumor cells may not always predict tumor progression or of its growth, invasion and metastasis and overall prognosis [12]. Therefore, it is imperative that other independent predictors of tumor progression be established.

In Japan, NETs mostly originate in the hindgut, with a relatively well-established endoscopic procedure used in most of these cases to remove these hindgut NETs [4, 13]. Previous study [8] has reported an overall prevalence of GI-NETs of 6.42 per 100,000 people (95 \% confidence interval (CI), 4.50 to 8.34). The specific prevalence rates of foregut, midgut, and hindgut NETs were estimated at 1.67 (95\% CI, 0.94-2.40), 0.23 (95\% CI, 0.18-0.28), and 4.52 (95\%, CI 3.17-5.87) per 100,000 people, respectively. In Japan, hindgut NETs account for approximately $70 \%(70.4 \%)$ of all GI-NETs (Table 1). Therefore, the aim of our retrospective study was to perform histopathological and statistical analyses of hindgut NETs, obtained by endoscopic procedures, to identify independent predictors of tumor progression and prognosis, based on pathological findings.

\section{Methods}

\section{Identification of hindgut NETs cases for analysis}

We searched for cases of hindgut NETs, recorded between April 1996 and September 2015, using a pathological diagnosis support software ('EXpath' System, INTEC Inc., Tokyo, Japan). The following keywords were used for the search: 'carcinoid,' 'neuroendocrine,' 'karuchinoid' (Japanese for carcinoid), and 'shinkeinaibunpi' (Japanese for

Table 1 Epidemiology of gastrointestinal neuroendocrine tumors in Japan (per 1000,000 population)

\begin{tabular}{ll}
\hline 2010 (JAPAN) & \\
\hline Overall prevalence of GI-NETs & $6.42(95 \% \mathrm{Cl} 4.50-8.34)$ \\
Foregut & $1.67(95 \% \mathrm{Cl} 0.94-2.40)$ \\
Midgut & $0.23(95 \% \mathrm{Cl} 0.18-0.28)$ \\
Hindgut & $4.52(95 \% \mathrm{Cl} 3.17-5.87)$ \\
Incidence of Gl-NETs & $3.51(95 \% \mathrm{Cl} 2.50-4.53)$ \\
Foregut & $1.20(95 \% \mathrm{Cl} 0.48-1.91)$ \\
Midgut & $0.15(95 \% \mathrm{Cl} 0.12-0.18)$ \\
Hindgut & $2.12(95 \% \mathrm{Cl} 1.56-2.67)$ \\
\hline
\end{tabular}

Legend: The foregut included the esophagus, stomach and duodenum; the midgut, the jejunum, ileum and vermiform appendix; and the hindgut, the large intestine and colon

GI-NET, gastrointestinal-neuroendocrine tumor; $95 \% \mathrm{Cl}, 95 \%$ confidence interval neuroendocrine). The terms 'ohkokettyou, Sjoketyou and tyokutyou, and S' (Japanese for transverse colon, sigmoid colon, and rectum) were used as an additional option to identify the tumor site. We subsequently retrieved the formalin-fixed paraffin-embedded (FFPE) tissue sections of identified hindgut NET cases.

\section{Clinicopathological data of identified hindgut NET cases}

We extracted the following clinical data from the medical records of identified hindgut NET cases for analysis: age, sex, and outcome. We also extracted reports of pathological findings for review. To conduct the pathological assessment of retrieved specimens, tissue sections from tumors were prepared and subjected to Hematoxylin and Eosin (HE) staining for analysis under a light microscope. Immunohistochemical examinations were performed using antibodies against the following markers: CD31 (Leica, clone 1A10; 1:20 dilution), chromogranin A (Roche, clone LK2H10; 1:5 dilution), D2-40 (Roche, clone D2-40; 1:1 dilution), Ki-67 (Dako, clone MIB-1; 1:50 dilution), and synaptophysin (Roche, clone MRQ-40; 1:1 dilution). Tumors were defined as NETs if immunoreactivity staining was positive for synaptophysin and/or chromogranin A. The Ki-67 labeling index was obtained by counting at least 1000 cells in each case, using the Patholoscope image analysis software (MITANI Corporation, Japan, URL: http://www.mitani-visual.jp/en/products/bio_imaging_analysis/patholoscope/).

For analysis, microscope images were captured at the center of the tumor primarily under two magnifications. Low and High power fields. However, other magnifications $(\times 100$ or $\times 200)$ were used for certain cases to produce multi-panel figures. This was necessary due to the relatively small number of specimens available for analysis and the absence of burtons surrounding stromal tissues. A scaling bar was included on all images to explain length measurements (and these are provided in the Figure legends). We examined the following additional pathological data as surrogate measures of tumor progression: tumor site, maximum tumor diameter, depth of tumor invasion, surgical margin status, and the status of lymph node metastasis.

Based on our identification of variation in the density of vessels and lymphatic vessels during histopathological examination of our cases, we selected to specifically calculate the microvessel density (MVD) and lymphatic microvessel density (LMVD) values in tumor specimens. MVD is defined as the number of blood vessels per unit area of tumor tissue, while LMVD refers to the number of lymphatic vessels per unit area. Previous investigators have reported that MVD may be one of the prognostic factors of the NETs [14].

To obtain these vessel counts, immunohistopathological images of the tumor stained for CD31 (for MVD 

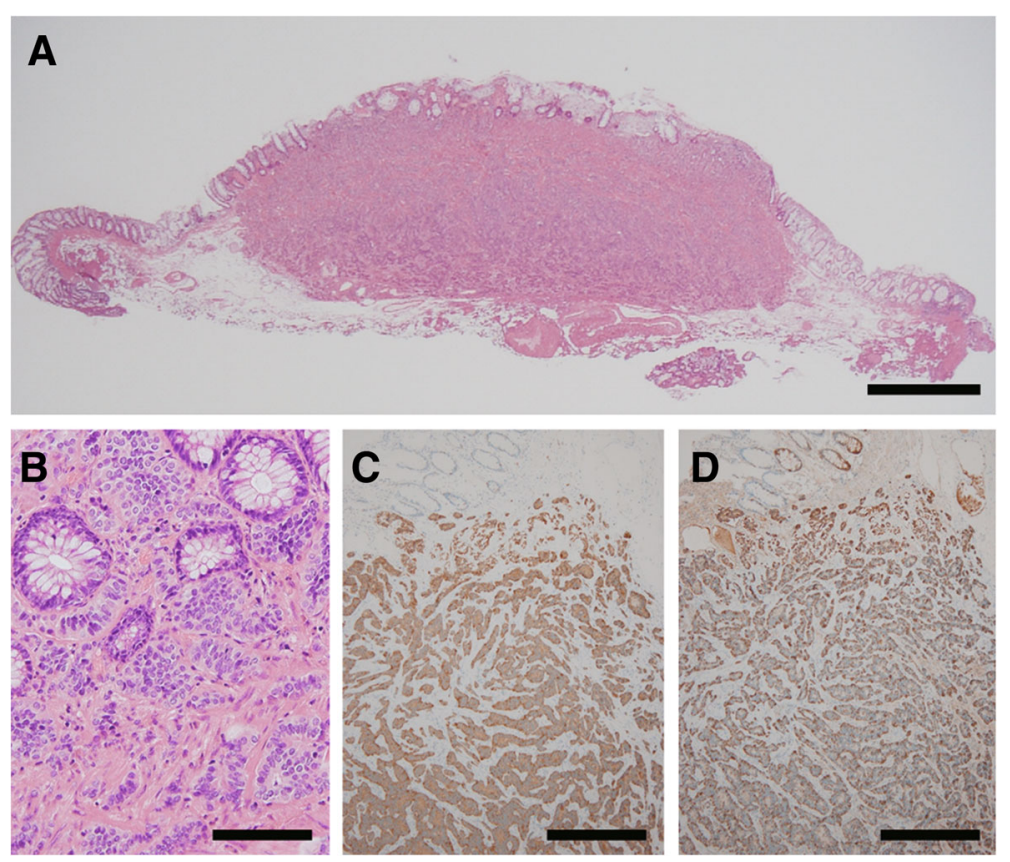

Fig. 1 Representative histopathological findings of hindgut neuroendocrine tumors. Legend: a A photomicrograph showing a low-power field of a hindgut neuroendocrine tumor (NET), with evidence of invasion of the submucosal layer (hematoxylin and eosin (HE) staining; original magnification, $\times 20$; scale bar represents $1000 \mu \mathrm{m}$ ). b A photomicrograph showing a high-power field of a hindgut NET. The tumor cells have a round-to-oval nucleus, and the nuclear atypia is relatively mild (HE staining; original magnification, $\times 400$; scale bar represents $100 \mu \mathrm{m}$ ). $\mathbf{c}$ and $\mathbf{d}$ Among the 42 cases in our study, a positive immunoreactivity for synaptophysin was identified in 42 cases and for chromogranin A in 35 cases (immunohistochemistry, synaptophysin and chromogranin A; original magnification, $\times 100$; scale bars represent $200 \mu \mathrm{m}$ )
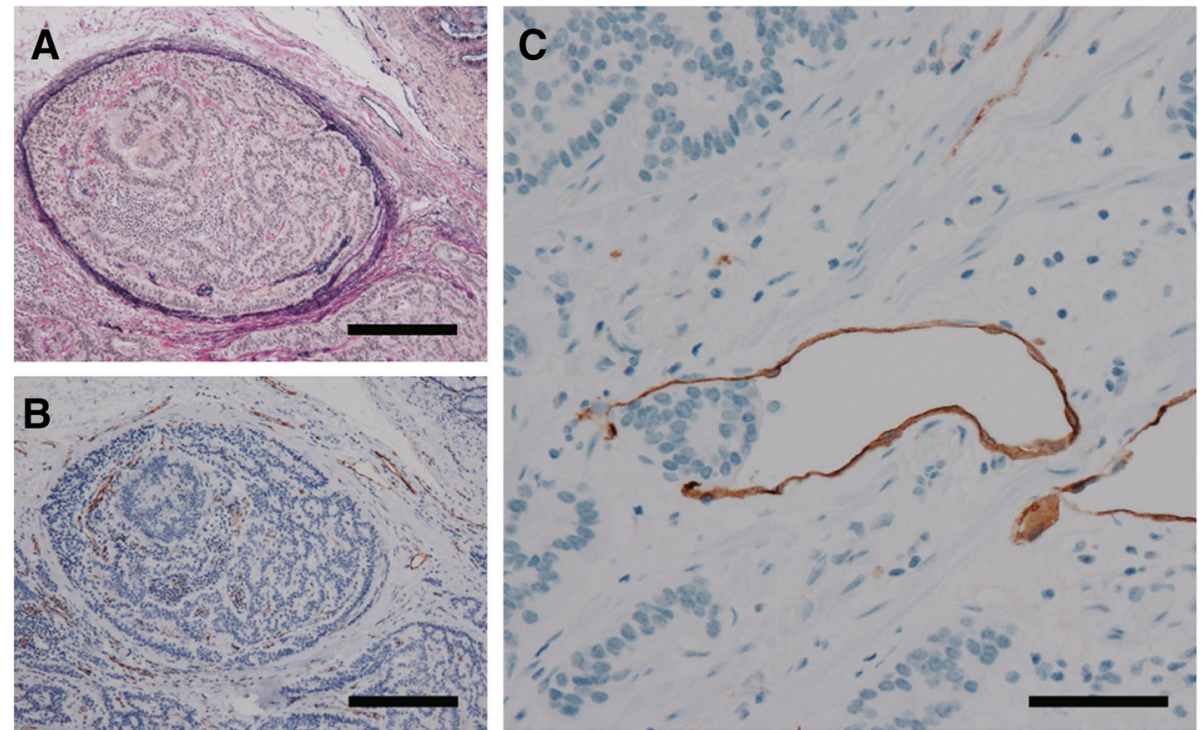

Fig. 2 Representative images of venous and lymphatic invasion. Legend: $\mathbf{a}, \mathbf{b}$ To determine the presence or absence of venous invasion, both EVG staining and immunohistochemistry for CD31 were performed. Venous invasion was confirmed, but no CD31-positive cells were identified. Such difficult cases were reviewed by more than two expert pathologists (EVG staining and immunohistochemistry for CD31; original magnification, $\times 200$; scale bars represent $200 \mu \mathrm{m})$. c To determine the presence or absence of lymphatic invasion, immunohistochemistry for D2-40 was performed. Difficult cases were examined by more than two expert pathologists (Immunohistochemistry for D2-40; original magnification × 400; scale bar represents $100 \mu \mathrm{m}$ ) 

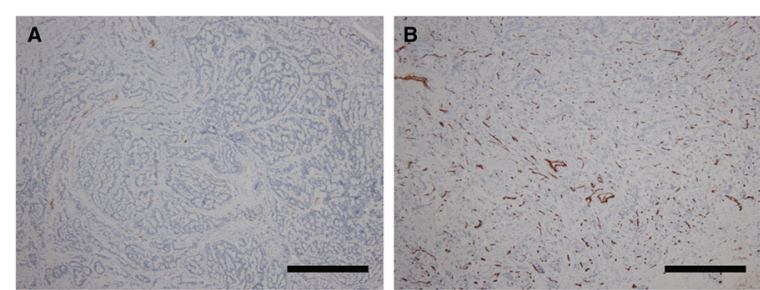

Fig. 3 Variation in microvessel density among tumors. Legend: a In this tumor, there are few CD31-positive endothelial cells and the tumor has an extremely low microvessel density. No venous invasion was observable (immunohistochemistry for CD31; original magnification, $\times 100$, scale; bar represents $100 \mu \mathrm{m})$. b In this tumor, numerous CD31positive endothelial cells were observable and the tumor has a high microvessel density. This is representative of most of cases with venous invasion (immunohistochemistry for CD31; original magnification, $\times 100$; scale bar represents $100 \mu \mathrm{m})$

count) or D2-40 (for LMVD count) were captured using a video microscope camera (DS-Fi1, Nikon, Tokyo, Japan), and the count carried out manually. CD31- and D2-40-positive structures were defined as vessels if a luminal structure was identified; single cells, positive for CD31 or D2-40, were not included. MVD and LMVD were counted in $20 \mathrm{HPFs}$ of histopathological images, using the hot spot counting method. Counting was facilitated by the specificity of CD31 for vascular endothelial cells, which is higher than when using CD34. For LMVD, although we recognized the low specific of D240 for lymphatic vessels, we used this staining technique because of its simplicity and instrumentation, as recognized by the Japanese Society for Cancer of the Colon and Rectum (URL:http://www.kanehara-shuppan.co.jp/ books/detail.html?isbn=9784307203142; in Japanese).

\section{Statistical analyses}

Appropriate statistical analyses were performed on the extracted data. Statistical analyses were performed using the non-parametric Mann-Whitney $U$ test or Pearson's product-moment correlation coefficient, as appropriate for the data set. Differences were considered significant at $P<0.05$.

\section{Results}

\section{Hindgut NETs cases included in the analysis}

Forty-seven relevant hindgut NET cases were identified, with specimens available for retrieval and analysis. Of these 47 specimens, a tumor lesion was identified in 42 of the collected FFPE tissue sections, with insufficient

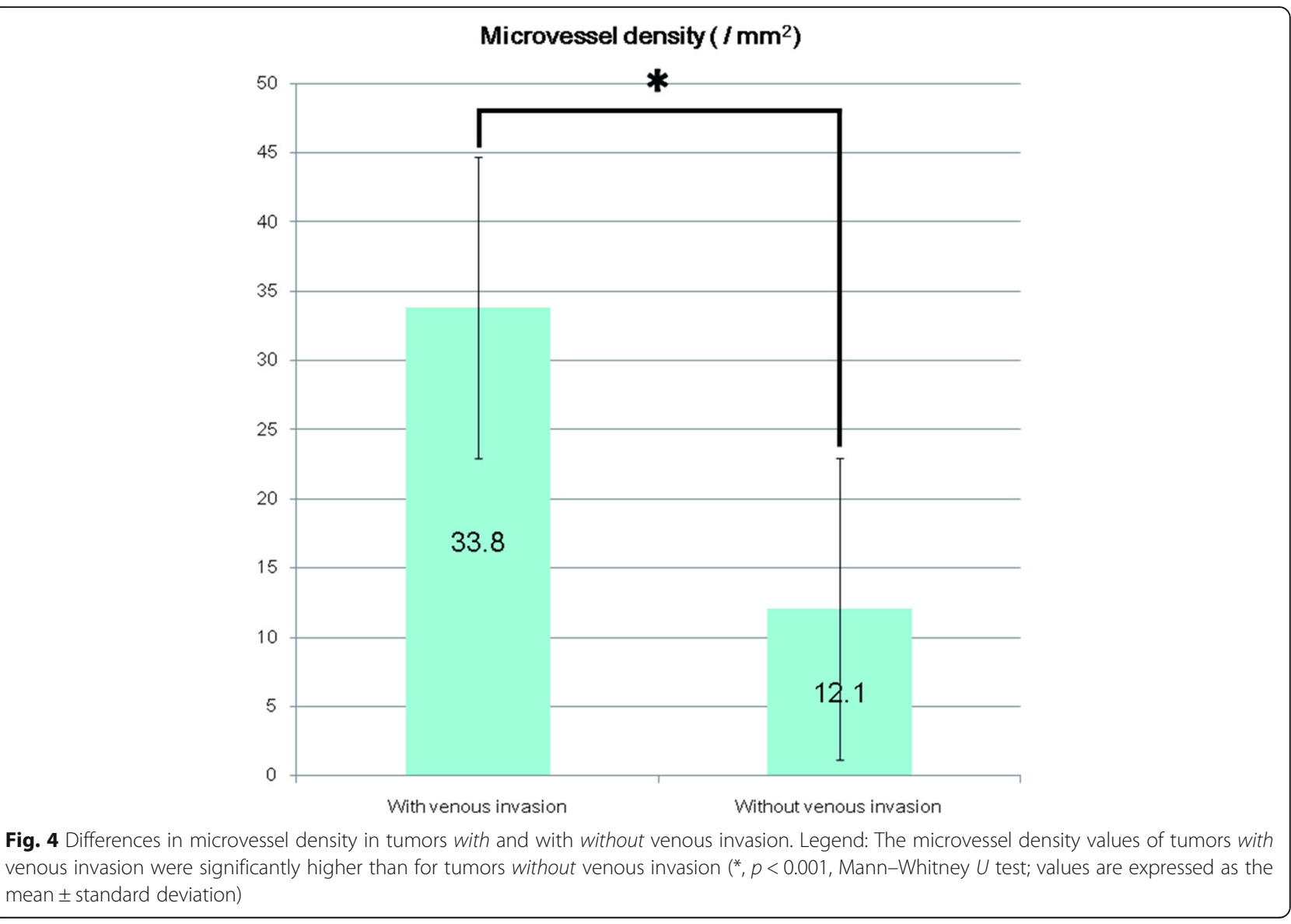


tumor tissue available for examination in the remaining 5 cases. The analysis was, therefore, based on the histopathological data of 42 cases of hindgut NETs.

\section{Clinicopathological data of hindgut NET cases}

The relevant clinical data were as follows: a mean \pm standard deviation (SD) of age of $59.6 \pm 12.0$ years (range, 37 to 81 years) and a male-to-female ratio of 23:19. No deaths from hindgut NETs were reported over the follow-up period, which ranged between 5 and 191 months. Four patients with hindgut NET underwent further surgical intervention due to lymphatic invasion detected in the specimen obtained during the initial endoscopic procedure. In 1 of these 4 patients, lymph node metastasis occurred in a level 1 lymph node. A positive surgical margin (vertical margin positive) was identified in another case, the patient refusing additional surgical intervention, but rather opting for observation and careful follow-up. This patient maintained a high quality of life over his 36 months of follow-up, after the endoscopic procedure, with no evidence of recurrent local or distant metastases. Four patients died of other disease causes, 2 of gastric cancer, 1 of lung cancer and 1 of cerebral hemorrhage. Overall, among the 42 cases of NETs, clinical characteristics were available in the medical record for only 22 patients who underwent regular medical follow-ups after the endoscopic procedure. With regards to pathological findings, 41 of the 42 cases of hindgut NETs occurred in the rectum and 1 in the sigmoid colon. The maximum diameter of the tumors ranged between 998.1 and $10046.0 \mu \mathrm{m}$ (mean \pm SD, $5058.0 \pm 2410.3 \mu \mathrm{m}$ ). The depth of tumor invasion was the submucosal layer in all 42 cases (Figs. 1a and b). On immunohistochemical examination, positive immunoreactivity for synaptophysin was identified in all 42 cases (100\%) and for chromogranin A was in 35 of 42 (83.3 \%) cases (Fig. 1c and d). The Ki-67 labeling index ranged between 0.1 and $3.2 \%$ (mean \pm SD, $1.1 \pm 0.8 \%$ ). On the basis of the Ki-67 labeling index, 34 cases were classified as NET G1 tumors and 8 as NET G2 tumors. Venous invasion was identified in 10 of 42 cases (23.8 \%) and lymphatic invasion in 13 of 42 cases (31.0 \%; Fig. 2). MVD and LMVD values varied among tumors (Fig. 3). MVD ranged between $1.4 / \mathrm{mm}^{2}$ and $73.9 / \mathrm{mm}^{2}$ (mean $\pm \mathrm{SD}, 17.3 \pm 14.2$ / $\mathrm{mm}^{2}$, Fig. 4) and LMVD from $0 / \mathrm{mm}^{2}$ to $22.9 / \mathrm{mm}^{2}$ (mean $\pm \mathrm{SD}, 6.5 \pm 6.5 / \mathrm{mm}^{2}$, Fig. 5). These data are summarized as Table 1.

\section{Statistical analyses findings}

To identify independent predictors of tumor progression, such as invasion and/or metastasis in hindgut NETs, we evaluated the predictive valued of MVD and LMVD. MVD values were higher in tumors with venous invasion (mean $\pm \mathrm{SD}, 33.8 / \mathrm{mm}^{2} \pm 20.7 / \mathrm{mm}^{2}$ than in tumors without venous invasion $\left(12.1 / \mathrm{mm}^{2} \pm\right.$

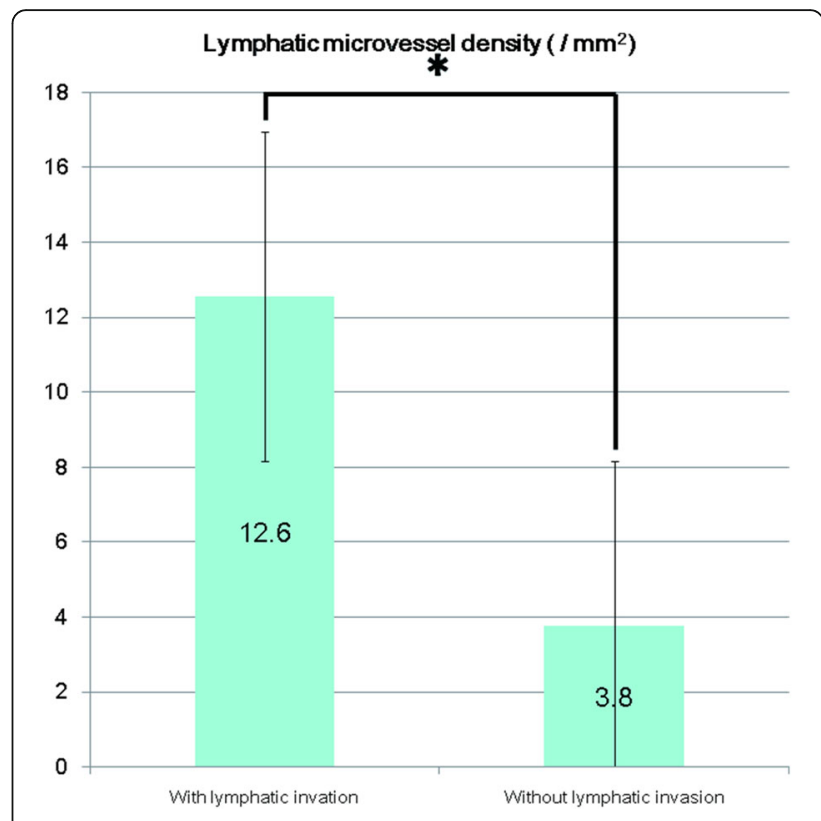

Fig. 5 Differences in lymphatic microvessel density in tumors with and without lymphatic invasion. Legend: The lymphatic microvessel density values of tumors with lymphatic invasion were significantly higher than for tumors without lymphatic invasion $\left({ }^{*}, p<0.001\right.$, Mann-Whitney $U$ test; values are expressed as the mean \pm standard deviation)

5.3/ $\mathrm{mm}^{2} ; p<0.001$, Mann-Whitney $U$ test; Fig. 4). Moreover, a positive correlation was identified between MVD and the maximum diameter of the tumor $(r=0.612, p<0.001$, Pearson's product-moment correlation coefficient; Table 2). Additionally, LMVD values were higher in tumors with lymphatic invasion than in tumors without lymphatic invasion $\left(12.6 / \mathrm{mm}^{2} \pm\right.$ $6.9 / \mathrm{mm}^{2}$ versus $3.8 / \mathrm{mm}^{2} \pm 4.1 / \mathrm{mm}^{2)} ; p<0.001$, MannWhitney $U$ test; Fig. 5). No significant correlation was identified between LMVD and the Ki-67 labeling index values or the maximum tumor diameter $(p=$ 0.744 and $p=0.136$, respectively, Pearson's productmoment correlation coefficient; Table 2). Due to the small number of cases, only univariate analyses of MVD and LMVD could be performed, and effects of age, grading, and sex could not be specifically evaluated.

\section{Discussion}

In this study, we specifically calculated the MVD and LMVD to confirm the presence or absence of lymphovascular invasion in hindgut NET cases, and performed statistical analyses to examine the relationship between these values and the Ki-67 labeling index and the maximum tumor diameter. Morphometric analysis further revealed MVD and LMVD to be risk factors of venous and lymphatic invasion in the initial phase of hindgut NETs. This latter finding results is explained by the 
Table 2 Clinicopathological chracteristics of hindgut neuroendocrine tumor

\begin{tabular}{|c|c|}
\hline \multicolumn{2}{|l|}{ Age (years) } \\
\hline Range & 37 to 81 \\
\hline mean $\pm S D$ & $59.6 \pm 12.0$ \\
\hline \multicolumn{2}{|l|}{$\mathrm{MVD}\left(\mathrm{mm}^{2}\right)$} \\
\hline Range & 1.4 to 73.9 \\
\hline mean $\pm S D$ & $17.3 \pm 14.2$ \\
\hline $\begin{array}{l}\text { Relationship to } \\
\text { venous invasion }\end{array}$ & $\begin{array}{l}\text { Significant positive correlation } \\
\text { (Mann-Whitney } U \text { test) }\end{array}$ \\
\hline \multicolumn{2}{|l|}{ LMVD $\left(\mathrm{mm}^{2}\right)$} \\
\hline Range & 0 to 22.9 \\
\hline mean $\pm S D$ & $6.5 \pm 6.5$ \\
\hline $\begin{array}{l}\text { Relationship to } \\
\text { lymphatic invasion }\end{array}$ & $\begin{array}{l}\text { Significant positive correlation } \\
\text { (Mann-Whitney } U \text { test) }\end{array}$ \\
\hline \multicolumn{2}{|l|}{ Gender (n, \%) } \\
\hline Male & $23,54.8 \%$ \\
\hline Female & $19,45.2 \%$ \\
\hline \multicolumn{2}{|l|}{ Pathological grade (n, \%) } \\
\hline NET G1 & $34,81 \%$ \\
\hline NET G2 & $8,19 \%$ \\
\hline \multicolumn{2}{|l|}{ Ki 67 labeling index (\%) } \\
\hline Range & 0.1 to 3.2 \\
\hline mean $\pm S D$ & $1.1 \pm 0.8$ \\
\hline $\begin{array}{l}\text { Relationship to tumor } \\
\text { diameter }\end{array}$ & $\begin{array}{l}\text { No significant correlation (Pearson's product- } \\
\text { moment correlation coefficient, } p=0.136 \text { ) }\end{array}$ \\
\hline \multicolumn{2}{|l|}{ Venous invasion (n, \%) } \\
\hline Negative & $32,76.2 \%$ \\
\hline Positive & $10,23.8 \%$ \\
\hline \multicolumn{2}{|l|}{ Lymphatic invasion (n, \%) } \\
\hline Negative & $29,69 \%$ \\
\hline Positive & $13,31 \%$ \\
\hline \multicolumn{2}{|l|}{ Tumor diameter ( $\mu \mathrm{m})$} \\
\hline Range & 998.1 to 10046.0 \\
\hline mean $\pm S D$ & $5058.0 \pm 2410.3$ \\
\hline Relationship to MVD & $\begin{array}{l}\text { Significant positive correlation } \\
\text { (Pearson's product-moment correlation } \\
\text { coefficient, } r=0.612, p<0.001 \text { ) }\end{array}$ \\
\hline Relationship to LMVD & $\begin{array}{l}\text { No significant correlation (Pearson's product- } \\
\text { moment correlation coefficient, } p=0.744 \text { ) }\end{array}$ \\
\hline \multicolumn{2}{|c|}{ Pathological stage $(n, \%)$} \\
\hline pStage I & $40,95.2 \%$ (all of them are $\mathrm{pT} 1$ and n0) \\
\hline pStage II & $0,0 \%$ \\
\hline pStage IIIB & 1, 2,4 \% (lymph node metasis positive) \\
\hline Unknown & 1, $2.4 \%$ (margin positive) \\
\hline
\end{tabular}

Legend: In this table clinicopathological characteristics and some statistical analyses of 42 hindgut neuroendocrine tumors were summarized MVD microvessel density, $L M V D$ lymphatic microvessel density, $S D$ standard deviation nature of our samples, with the maximum tumor diameter being $<10 \mathrm{~mm}$ in most cases.

Our findings provide evidence on the relationship between tumor progression and angiogenesis. Although it is widely accepted that the Ki-67 labeling index generally reflects tumor progression $[15,16]$, we did not identify a significant correlation between the Ki-67 labeling index and the maximum tumor diameter. In contrast, we did identify a significant positive correlation between MVD and maximum tumor diameter, indicative of possible common mechanisms underlying NET progression and angiogenesis in GEP-NETs. In addition, as venous invasion of NETs is a risk factor of metastasis, this mechanism could influence not only the progression of NETs but also the spread of distant metastases (especially liver metastasis). Our analyses revealed a significant positive correlation between the maximum tumor diameter and MVD. Previous investigators have also reported that MVD may be one of the prognostic factors of NETs [14]. The plausible effect of angiogenesis on tumor progression is further supported by absence of a correlation between the LMVD and Ki-67 labeling index values or the maximum tumor diameter.

Based on this evidence of a plausible effect of angiogenesis on tumor progression, novel angiogenesis targeting agents could prove beneficial in the treatment of NETs. Future research should include molecular, biological, and genetic analyses, such as the angiogenesisrelated genes, to more comprehensively identify novel independent factors of tumor progression, as well as to inform the development of new, and likely more effective, treatment strategies.

The limitations of our study need to be considered in the interpretation of our results. Foremost, this is a retrospective case series and, therefore, is subject to the inherent biases of this research design. Moreover, our analysis was based on only 42 cases of NETs, which prohibited the use of multivariate analyses to further explore the predictive effects of age, sex, and tumor staging, among other relevant clinicopathological characteristics.

Despite these limitations, our study provides evidence of a plausible role of angiogenesis in the occurrence and progression in the initial phase of hindgut NETs. Our findings provide a basis for future studies examining the role of angiogenesis-related genes and of targeted gene therapies as novel treatments for NETs.

\section{Conclusions}

Our study indicated that angiogenesis mechanism play important roles in occurrence and progression in the initial phase of hindgut NETs. Furthermore, our data indicated that molecular, biological, and genetic analyses, such as the examination of angiogenesis-related genes, might provide efficient and new research strategies to 
elucidate the progression of NETs as well as identify novel independent predictors of these tumors.

\section{Abbreviations}

GEP-NET: Gastroenteropancreatic neuroendocrine tumor; WHO: World Health Organization; HPF: High-power field; FFPE: Formalin-fixed paraffin-embedded; HE: Hematoxylin and Eosin; MVD: Microvessel density; LMVD: Lymphatic microvessel density; SD: Standard deviation

\section{Acknowledgements}

The authors also thank to Sachie Osanai and Rie Kajihara for their excellent pathological and technical support and Keisuke Kazama, Tetsuta Satoyoshi, Atsushi Wada, and Takao Ishiwatari for their clinicopathological advice.

\section{Funding}

This work was supported by a Grant-in-Aid for Scientific Research (\#26860774) from the Ministry of Education, Culture, Sports, Science, and Technology of Japan and a research grant from the Kanagawa Cancer Center (\#Heisei28-07).

\section{Availability of data and materials}

The dataset supporting the conclusions of this article is included within the article (Figs. 1, 2, 3, 4 and 5 and Tables 1 and 2). In addition, all measurements were collected and recorded in Microsoft Excel and slides are stored in the Surgical Pathology archives at Kanagawa Cancer Center. All material is available upon request.

\section{Authors' contributions}

YO conceptualized this study, integrated the data, performed statistical evaluation, and wrote the manuscript. OM, NN, and KN performed endoscopic procedures and extracted clinical data from the electronic medical record system of our institute. RK and YM performed a part of the morphometric analyses of MVD/LMVD and performed the statistical analyses. MS performed operation and advised the first author as the chief doctor of gastroenterological surgery. EY, MS, KW, KK, and MN integrated the clinicopathological data of patients with the tumor characteristics, and performed a part of the histopathological examinations. YK and TY performed a part of the histopathological examinations, integrated the data and revised the manuscript. All authors read and approved the final manuscript.

\section{Competing interests}

The authors declare that they have no competing interests.

\section{Consent for publication}

Not applicable.

\section{Ethics approval and consent participate}

This retrospective study was performed in accordance with the Declaration of Helsinki and approved by the Ethics Review Committee of Kanagawa Cancer Center, Kanagawa, Japan (Approval Number: 27-38). Furthermore, a general consent was obtained at the time of clinical intervention for the future use of material for research in all cases.

\section{Author details}

'Department of Pathology, Kanagawa Cancer Center, 2-3-2, Nakao, Asahi-Ku Yokohama, Kanagawa 241-8515, Japan. ${ }^{2}$ Department of Gastroenterology, Kanagawa Cancer Center, 2-3-2, Nakao, Asahi-Ku, Yokohama, Kanagawa 241-8515, Japan. ${ }^{3}$ Molecular Pathology and Genetics Division, Kanagawa Cancer Center Research Institute, 2-3-2, Nakao, Asahi-Ku, Yokohama, Kanagawa 241-8515, Japan. ${ }^{4}$ Department of Gastrointestinal Surgery, Kanagawa Cancer Center, 2-3-2, Nakao, Asahi-Ku, Yokohama, Kanagawa 241-8515, Japan. ${ }^{5}$ Department of Thoracic Surgery, Kanagawa Cancer Center, 2-3-2, Nakao, Asahi-Ku, Yokohama, Kanagawa 241-8515, Japan.

Received: 6 August 2016 Accepted: 3 November 2016 Published online: 08 November 2016

\section{References}

1. Cho MY, Kim JM, Sohn JH, Kim MJ, Kim KM, Kim WH, Kim H, Kook MC, Park Do Y, Lee JH, Chang H, Jung ES, Kim HK, Jin SY, Choi JH, Gu MJ, Kim S,
Kang MS, Cho CH, Park MI, Kang YK, Kim YW, Yoon SO, Bae HI, Joo M, Moon WS, Kang DY, Chang SJ. Current Trends of the Incidence and Pathological Diagnosis of Gastroenteropancreatic Neuroendocrine Tumors (GEP-NETs) in Korea 2000-2009: Multicenter Study. Cancer Res Treat. 2012;44:157-65.

2. Palmieri F, Brunocilla E, Bertaccini A, Guidi M, Pernetti R, Morselli-Labate AM, Martorana G. Prognostic value of lymphovascular invasion in bladder cancer in patients treated with radical cystectomy. Anticancer Res. 2010;30:2973-6.

3. Schoppmann SF, Bayer G, Aumayr K, Taucher S, Geleff S, Rudas M, Kubista E, Hausmaninger H, Samonigg H, Gnant M, Jakesz R, Horvat R. Prognostic value of lymphangiogenesis and lymphovascular invasion in invasive breast cancer. Ann Surg. 2004;240:306-12.

4. Nakamura K, Osada M, Goto A, Iwasa T, Takahashi S, Takizawa N, Akahoshi K Ochiai T, Nakamura N, Akiho H, Itaba S, Harada N, lju M, Tanaka M, Kubo H, Somada S, Ihara E, Oda Y, Ito T, Takayanagi R. Short- and long-term outcomes of endoscopic resection of rectal neuroendocrine tumours: analyses according to the WHO 2010 classification. Scand J Gastroenterol. 2016;51:448-55.

5. Stinner B, Rothmund M. Neuroendocrine tumours (carcinoids) of the appendix. Best Pract Res Clin Gastroenterol. 2005;19:729-38.

6. Griniatsos J, Michail O. Appendiceal neuroendocrine tumors: Recent insights and clinical implications. World J Gastrointest Oncol. 2010;2:192-6.

7. Okubo Y, Wakayama M, Nemoto T, Kitahara K, Nakayama H, Shibuya K, Yokose T, Yamada M, Shimodaira K, Sasai D, Ishiwatari T, Tsuchiya M, Hiruta N. Literature survey on epidemiology and pathology of gangliocytic paraganglioma. BMC Cancer. 2011;11:187.

8. Ito T, Igarashi H, Nakamura K, Sasano H, Okusaka T, Takano K, Komoto I, Tanaka M, Imamura M, Jensen RT, Takayanagi R, Shimatsu A. Epidemiological trends of pancreatic and gastrointestinal neuroendocrine tumors in Japan: a nationwide survey analysis. J Gastroenterol. 2015;50:58-64

9. Oberg K, Castellano D. Current knowledge on diagnosis and staging of neuroendocrine tumors. Cancer Metastasis Rev. 2011;30 Suppl 1:3-7.

10. Oberg KE. Gastrointestinal neuroendocrine tumors. Ann Oncol. 2010;21 Suppl 7:vii72-80.

11. Okubo Y, Nemoto T, Wakayama M, Tochigi N, Shinozaki M, Ishiwatari T, Aki K, Tsuchiya M, Aoyama H, Katsura K, Fujii T, Nishigami T, Yokose T, Ohkura Y, Shibuya K. Gangliocytic paraganglioma: a multi-institutional retrospective study in Japan. BMC Cancer. 2015:15:269.

12. Zimmermann ME, Bosman FT. Proliferative activity of well differentiated neuroendocrine tumours of the gut. Histol Histopathol. 2003:18:353-8.

13. Nemoto D, Hayashi Y, Utano K, Isohata N, Endo S, Lefor AK, Yamamoto H, Togashi K. A novel retrieval technique for large colorectal tumors resected by endoscopic submucosal dissection: tumor extraction by defecation. Endosc Int Open. 2016;4:E93-5.

14. Kuiper $P$, Hawinkels $L$, de Jonge-Muller $E S$, Biemond I, Lamers $C B$ Verspaget HW. Angiogenic markers endoglin and vascular endothelial growth factor in gastroenteropancreatic neuroendocrine tumors. World J Gastroenterol. 2011;17:219-25.

15. Vilar E, Salazar R, Perez-Garcia J, Cortes J, Oberg K, Tabernero J. Chemotherapy and role of the proliferation marker Ki-67 in digestive neuroendocrine tumors. Endocr Relat Cancer. 2007:14:221-32.

16. Okubo Y, Shibuya K, Namiki A, Takamura K, Kameda N, Nemoto T, Mitsuda A, Wakayama M, Shinozaki M, Hiruta N, Kitahara K, Ishiwatari T, Yamazaki J. Leiomyosarcoma with partial rhabdomyoblastic differentiation: first case report of primary cardiac origin. BMC Cancer. 2011;11:76.

\section{Submit your next manuscript to BioMed Central and we will help you at every step:}

- We accept pre-submission inquiries

- Our selector tool helps you to find the most relevant journal

- We provide round the clock customer support

- Convenient online submission

- Thorough peer review

- Inclusion in PubMed and all major indexing services

- Maximum visibility for your research

Submit your manuscript at www.biomedcentral.com/submit
Biomed Central 